\title{
Utilizing Continuous Flow Microdevices-based Magnetophoresis for COVID-19 Diagnosis
}

\author{
Sawsan Dagher 1,*(D), Ali Hilal-Alnaqbi ${ }^{2 \mathbb{C}}$, Boshra Akhozheya ${ }^{3(\mathbb{1})}$, Laila Akhu-Zaheya ${ }^{4(\mathbb{C})}$ \\ 1 Electromechanical Engineering Technology, Abu Dhabi Polytechnic, Abu Dhabi, UAE; sawsan.dagher@adpoly.ac.ae \\ (S.D.); \\ 2 Abu Dhabi Polytechnic, Abu Dhabi, UAE; Ali.Alnaqb@adpoly.ac.ae (A.H.A.); \\ 3 Department of Building \& Architectural Engineering, Polytechnic University of Milan, Milan, Italy; \\ boshrakhaled.akhozheya@mail.polimi.it (B.A.); \\ 4 Department of Nursing, Jordan University of Science and Technology, Irbid, Jordan; lailanurs@just.edu.jo (L.A.Z.); \\ * Correspondence: sawsan.dagher@adpoly.ac.ae;
}

Received: 29.07.2020; Revised: 15.12.2020; Accepted: 22.12.2020; Published: 28.12.2020

\begin{abstract}
The contagious disease called COVID-19 is caused by a novel severe acute respiratory syndrome coronavirus 2 (SARS-CoV-2). Several precautionary and preventive measures are currently implemented, including travel restrictions, health quarantine, and medical care. However, no explicit treatments are available. Thus, a better understanding of the new infectious disease's nature and the contributing virus is required. This article will briefly review the biological structure and mechanism of the SARS-CoV-2, comparing it to other known coronaviruses, and the potential therapeutic approaches currently under investigation, and propose for the first time the utilization of microfluidic devices based magnetophoresis for SARS-CoV-2 detection and point of care diagnostics as a future potential effective method.
\end{abstract}

Keywords: SARS-CoV-2; coronavirus; COVID-19; magnetophoresis; microfluidic.

(C) 2020 by the authors. This article is an open-access article distributed under the terms and conditions of the Creative Commons Attribution (CC BY) license (https://creativecommons.org/licenses/by/4.0/).

\section{Introduction}

Since December 2019, the world has shown much attention towards the cumulative number of pneumonia patients, which existed in Wuhan, Hubei province, China, and currently spread across the world [1,2]. Huge efforts all over the world are put to prevent the virus outbreak among populations and to find a treatment for patients. World Health Organization (WHO) named the new pneumonia disease as Coronavirus Disease 19 (COVID-19). When first discovered, the virus was branded as 2019-nCoV or "novel coronavirus 2019", after a while, the virus was given a scientific term, severe acute respiratory syndrome coronavirus 2 (SARSCoV-2) [1,2].

Coronavirus belongs to the Nidovirales order and the family of Coronaviridae. The scientific name for coronavirus is Coronavirinae. The coronavirus subfamily is classified into four major genera: Alpha-coronavirus, Beta-coronavirus, Gamma-coronavirus, and Deltacoronavirus. The human coronaviruses (HCoVs) are in two of these genera, seven kinds of human coronaviruses have been identified, including HCoV-NL63 and HCoV-229E, which belong to the Alpha-coronavirus genus; and HCoV-OC43, HCoV-HKU1, severe acute respiratory syndrome coronavirus (SARS-CoV), Middle East respiratory syndrome coronavirus (MERS-CoV), and SARS-CoV-2, which all fit in the Beta-coronavirus genus [3- 
5]. The taxonomy of Coronaviridae, according to the International Committee on Taxonomy of Viruses, is shown in Figure 1.

Two Beta-coronaviruses SARS-CoV emerged in China in 2002. MERS-CoV emerged in the Arabian Peninsula in 2012 are zoonotic origin, that transmitted to human using bats/palm civets and dromedary camels. Presently, antiviral vaccines or treatments haven't yet been developed to cure humans affecting coronaviruses. Cross-species transmission of the coronaviruses is not expected to disappear. Future transmissions of the virus across species are yet to be expected due to the large reservoir found in bats [6,7]. Therefore, analyzing coronaviruses will support the comprehending of principles leading to cross-species spread and variation to humans. Conferring to the Center for Disease Control and Prevention (CDC), many viruses, especially RNA viruses, rapidly accumulate genetic variation because of short generation times and high mutation rates. Thus, it is a need to study the genome sequence of the new coronavirus to develop accurate diagnostic, antiviral drug, or therapy.

Furthermore, rapid and complete recognition and subtyping of the coronaviruses during the early stage of infection are required to deliver an instant and precise diagnosis and avert it is spread timely. Conventionally, several types of diagnostic approaches have been used to investigate COVID-19 infection, a clinical laboratory test to determine the type of virus has become a significant part of the epidemiological study, and epidemic inhibition. This study will discuss the principle of a recent method utilized in virus detection called magnetic microfluidic detection, opening the door to be applied for SARS-CoV-2 detection as a future potential effective approach $[8,9]$.

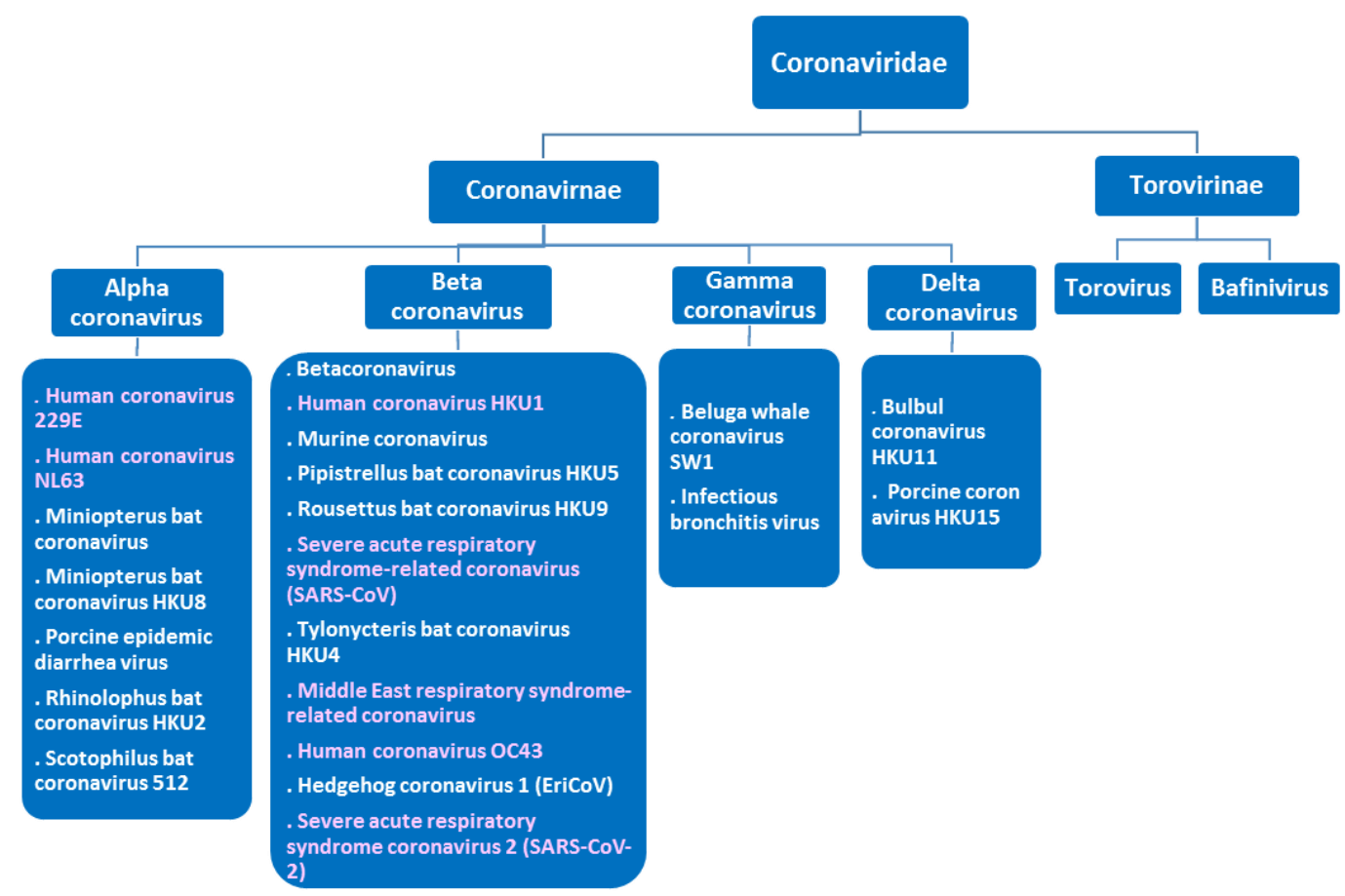

Figure 1. Taxonomy of the family of Coronaviridae.

\section{SARS-CoV-2 Structure}

Recent research showed that each SARS-CoV-2 virion is spherical in shape with 50$200 \mathrm{~nm}$ diameter. SARS-CoV-2, similar to other coronaviruses, is a big enclosed positivesense RNA virus, which consists of four structural proteins, three of them contribute to creating the viral envelope identified as the $\mathrm{S}$ (spike), E (envelope), $\mathrm{M}$ (membrane), and one protein is identified as the N (nucleocapsid) which binds the RNA genome as shown in Figure 2. When 
SARS-CoV-2 contaminates a cell; (i) the spike (S) protein in the viral envelope directly attaches to the angiotensin-converting enzyme 2 (ACE2) receptor on the host cell membrane (i.e., epithelial cells within the lungs); (ii) these spikes undergo a structural change (i.e., priming) onto human cells that allows the viral membrane to fuse with the cell membrane, facilitating virus entry and replication. SARS-CoV-2 uses an enzyme called TMPRSS2 for S protein priming; (iii) the RNA genome enters the host cell, splits into smaller segments, thus reducing the chance that an error in a single-component genome will disable the entire genome, then to be copied, producing more viruses [10,11].

The results obtained by investigating full-length of the SARS-CoV-2 genome sequences of five patients at an early stage of the disease outbreak showed that the five genomes are almost matching. The genome sequences of the SARS-CoV-2 and SARS-CoV are shared with $79.6 \%$. At the same time, it is showing a $96 \%$ similarity to a bat coronavirus with only $4 \%$ variability in the whole genomic nucleotides [12]. Although the genetic material of SARSCoV-2 and bat coronavirus is almost identical, there are substantial variations between a specific site known as the receptor-binding domain (RBD), the spike protein in the two viruses, the difference at these sites was $17 \%$. These spike variations in SARS-CoV-2 and bat coronavirus are likely initiated by mutations, natural selection, and recombination. The RBD is a key part of coronaviruses, which allows them to attach to and enter a cell. This proposes that the virus did not directly pass to humans from bats. Instead, it was transmitted to people through an intermediate host. Another study showed a 99\% genetic match between the RBD sites of SARS-CoV-2 and smuggled pangolins coronavirus [13].

Recently, research showed the genome sequence of SARS-CoV-2 of different patients in Brazil and Europe who have COVID-19 are different from each other. Moreover, it is different from the SARS-CoV-2 genomes samples sequenced in Wuhan, China. Later on, differences in the SARS-CoV-2 genomes are found, in which it is categorized into two types of the coronavirus (designated $\mathrm{L}$ and $\mathrm{S}), \mathrm{L}$ type $(\sim 70 \%)$ is more dominant and aggressive. It spreads more quickly than the $\mathrm{S}$ type $(\sim 30 \%)$ which is the initial version first detected in China. These results support the urgent need for more studies on the developed coronavirus L type $[13,14]$.

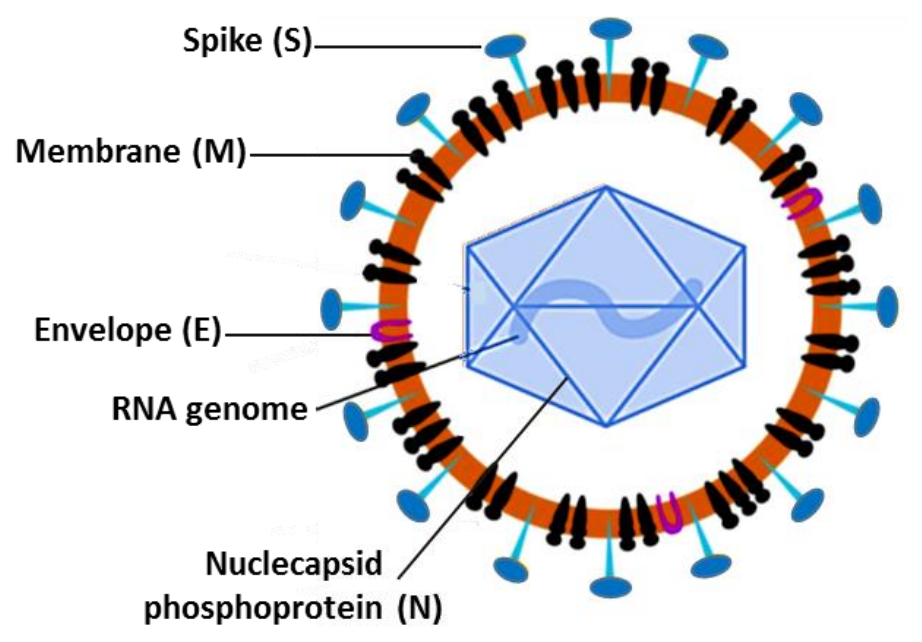

Figure 2. Schematic of the Biotic structure of the novel severe acute respiratory syndrome coronavirus 2 (SARS-CoV-2) [11]. "Reprinted from Journal of Infection, 80, Han Q.; Lin, Q.; Jin, S.; You, L., Coronavirus 2019-nCoV: A brief perspective from the front line, 374, 2020, with permission from Elsevier." 


\section{Characteristics of SARS-CoV, MERS-CoV, and SARS-CoV-2}

SARS-CoV, MERS-CoV, and SARS-CoV-2 all belong to the betacoronavirus genus, which infects the respiratory system. They have the same common major symptoms, which are fever, cough, shortness of breath, pneumonia. At the same time, they vary in severity, and other minor symptoms accompany. The person can be infected by respiratory droplets (droplet spread) produced when an infected person coughs or sneezes, when a person touches a surface or object contaminated with infectious droplets and then touches his or her mouth, nose, or eyes. A comparison between these three viruses is shown in Table 1.

MERS has the highest fatality rate but the least spread rate, while SARS-CoV-2 has the highest outbreak and the lowest mortality, with an increase in its criticality witnessed among older people and people with pre-existing medical conditions such as; cardiovascular disease, hypertension, chronic respiratory disease, diabetes, cancer.

The researchers found that SARS-CoV-2 spreads more easily from person to person than the earlier virus. Due to the fact that the SARS-CoV-2 spike has 10 to 20 times higher affinity to bind ACE2 on human cells than the spike from the SARS-CoV from 2002. On the other hand, SARS-CoV and MERS-CoV proteins bind to different RBD on different host receptors.

The sequence and structure of the spikes of the SARS-CoV from 2002 and SARS-CoV2 are similar. However, three different antibodies against the SARS-CoV from 2002 could not bind effectively to the SARS-CoV-2 spike protein. Thus, new treatment strategies for COVID91 is required by proposing a unique vaccine and antibody for the SARS-CoV-2 [15-19].

Table 1. Comparison between SARS-CoV, MERS-CoV, and SARS-CoV-2.

\begin{tabular}{|c|c|c|c|}
\hline & \multicolumn{3}{|c|}{ Coronavirus Strain } \\
\hline Characteristics & SARS-CoV & MERS-CoV & SARS-CoV-2 \\
\hline Detection date & November, 2002 & Jun, 2012 & December, 2019 \\
\hline Detection location & Guangdong, China & Saudi Arabia & Wuhan, China \\
\hline Cases & 8096 & 2494 & $15,406,223$ (July 23) \\
\hline Deaths (Mortality rate) & $774(10 \%)$ & $858(37 \%)$ & $631,030(4 \%)$ (July 23) \\
\hline Age average & 39.9 & 56 & 49 \\
\hline Age range & $1-91$ & $14-94$ & $21-76$ \\
\hline Male: Female ratio & $1: 1.25$ & $3.3: 1$ & $1.67: 1$ \\
\hline Healthcare practitioners & $23.1 \%$ & $9.8 \%$ & $29 \%$ \\
\hline Incubation period & 2-14 day & 2-15 day & 2-14 day \\
\hline Intermediate host & $\begin{array}{l}\text { Game food mammals } \\
\text { (civets and raccoon dogs } \\
\text { in southern China) }\end{array}$ & $\begin{array}{l}\text { Dromedary camels } \\
\text { (the Middle East } \\
\text { and Africa) }\end{array}$ & $\begin{array}{l}\text { Bats as the potential natural reservoir and } \\
\text { pangolins as the possible intermediate host of } \\
\text { the virus [2]. }\end{array}$ \\
\hline Viral phylogeny Lineage & B 2, 9 & $\mathrm{C}$ & $\mathrm{B}$ \\
\hline Host receptor & ACE2 & DPP4 (CD26) & ACE2 \\
\hline $\begin{array}{l}\text { Major host proteases that activate } \\
\text { spike protein }\end{array}$ & $\begin{array}{l}\text { Cathepsin L, TMPRSS2, } \\
\text { HAT }\end{array}$ & $\begin{array}{l}\text { TMPRSS2, } \\
\text { Cathepsin L, Furin }\end{array}$ & TMPRSS2 \\
\hline Dominant cell entry pathway & Endosomal fusion & $\begin{array}{l}\text { Cell membrane } \\
\text { fusion }\end{array}$ & Endosomal fusion \\
\hline Some antiviral drugs Therapy & $\begin{array}{l}\text { Chloroquine } \\
\text { Remdesivir }\end{array}$ & Remdesivir & $\begin{array}{l}\text { Researches in vitro: } \\
\text { Remdesivir } \\
\text { Chloroquine } \\
\text { Camostat mesylate, an inhibitor of TMPRSS2 }\end{array}$ \\
\hline
\end{tabular}

\section{Potential CQVID-19 Therapy}

Many approaches for COVID-19 therapy have been proposed and tested, some of them based on (i) improvement of the patients' immune system, (ii) antibodies made by people who had infected with SARS-CoV-2 and recovered. Antibodies are proteins produced by the body's immune system to attack and kill viruses, (iii) use of general available antiviral drugs, (iv) 
development of new drugs based on an understanding of the SARS-CoV2 genome structure, RPD, and its mechanism of entry to the host cell.

An in vitro study shows that Remdesivir and Chloroquine are highly effective in blocking SARS-CoV2 infection. They interfere with the glycosylation of cellular receptors (ACET2) to prevent SARS-CoV-2 virus entry into cells.

Another study finds that Camostat Mesylate, a drug used to treat pancreatitis, can reduce SARS-CoV-2 entry into cells by inhibiting the mechanism of the enzyme known as TMPRSS2 responsible for $\mathrm{S}$ protein priming. Thus membrane fusion is inhibited, which prevents a SARS-CoV-2 from being able to enter lung cells. Other clinical trials are being done as an attempt to reduce the symptoms faced by COVID-19 patients, which include: the use of a combination of two drugs namely; Lopinavir and Ritonavir, and the use of Favilavir, which is an antiviral drug that showed a significant reduction in the symptoms, however, none of these medications has been approved to treat the COVID-19 [20-22].

\section{Microfluidic Magnetic Devices for Virus Detection}

The most common protocols for diagnosing SARS-CoV-2 infection are (i) the polymerase chain reaction (PCR), a molecular test used to detect viral RNA in the sample by multistep nucleic acids extraction. (ii) Serological assays such as FIA and ELISA are utilized to spot previous infection (antibodies to SARS-CoV-2) in people who may have been subjected to an infected environment. This technique is based on detecting color change or fluorescencelabeled on antibodies involving a series of tedious processes such as incubation and washing steps; it takes over 4 hours. Both methods are labor-intensive and require well-trained technicians, relatively expensive instruments, and reagents, prohibiting on-site and real-time practical applications.

Therefore, the integration of nanotechnology along with microfluidic new methods that can overcome this problem, is urgently needed for the simultaneous and automated detection of multiple viruses' subtypes on the microfluidic chip. This method is based on miniaturization, which has unique advantages over conventional analytical techniques. Those advantages include reduced consumption of sample and reagent, which permits high throughput, convenient manipulation in very low volumes, high level of automation, enhanced surface area to volume ratio, and fast reaction times which greatly saves the detection time, and greatly improves the sensitivity. These characteristics make microfluidic chips suitable for process integration, such as; separation, enrichment, reaction, and detection of target viruses.

Different types of actuation forces, including magnetophoresis, have been employed in microfluidic devices to carry out separation with accurate identification from complex biological fluids extracts. Magnetophoresis refers to the movement of magnetic entities in response to a non-uniform magnetic field. Devices based on integrating magnetic functionality with microfluidics have been demonstrated, where functional magnetic nanoparticles are used to provide a large specific surface for chemical binding with target viruses in a sample solution. In recent years, magnetic nanomaterials have been extensively used to separate and enrich bacteria, viruses, and cells due to their unique properties such as high surface-to-volume, easy modification, high magnetic susceptibility, and low loss rate during the treatment process. Thus, many efforts have been devoted to developing microfluidic chips with magnetic nanoparticles to accomplish the detection and subtyping of multiple viruses such as influenza viruses (H1N1, H3N2, H9N2) simultaneously. These essays on microfluidic chips usually need fabrication methods like soft lithography, fine magnetic field manipulation [23-25]. 
Because the coronavirus was so new, the amount of data available on it is still small; we believe that utilizing microchips based magnetophoresis can allow us to discover innovative paths for simultaneous usage of different-size magnetic particles to detect multiple viruses' subtypes.

\subsection{Preparation of immune magnetic beads.}

The mobility of magnetic nanoparticles relative to the ambient fluid is based on two factors: the susceptibility mismatch between the magnetic nanoparticles and the surrounding fluid. The second is the gradient of the magnetic field. Thus, to obtain manipulation of magnetic nanoparticles (i.e., trapping, focusing, separation, sorting) based on magnetophoresis, the magnetic nanoparticles flow inside non-magnetic (e.g., water) surrounding medium are magnetized under external fields and therefore move towards the location of field maxima.

However, in order to magnetically manipulate viruses, using the magnetophoresis technique, modification of their magnetic properties is required. By attaching target viruses to magnetic nanoparticles; involves first magnetic nanoparticles to be transformed into immune magnetic beads (IMBs) via antibody modification, then labeling viruses with IMBs as shown in Figure 3, in which viruses and IMBs must be incubated together for several hours, then multiple washing steps are needed, prior to manipulation. One common procedure is by following these steps; (i) conjugation of biotin to antibodies; in which the antibodies are exposed to the biotin compound and allowed to react to form the biotin modified antibodies, the biotin solution and antibodies are incubated for 4 hours with continuous shaking to modify the biotin on the antibodies. (ii) Virus-antibodies are restrained on the surface of magnetic nanoparticles through the process of crosslinking the amines of the antibodies with the carboxylic acid groups; magnetic nanoparticles are transformed into IMB after antibody modification on their surface. (iii) Virus samples and IMBs are allowed to react and are injected into the microchannel at a certain flow rate, where the IMBs tagged virus are separated from a complex mixture. After that, the IMBs marked virus can be captured or sorted based on sizes [26].

After binding the magnetic nanoparticles of similar sizes to the SARS-CoV-2 biomarkers (i.e., IMBs), the sample that may contain the virus and the IMBs are both injected in the continuous flow microdevice, the IMBs that have the biomarker for SARS-CoV-2 viruses could attach it, to detect whether the sample has a virus and another approach to use it for a therapy analysis.

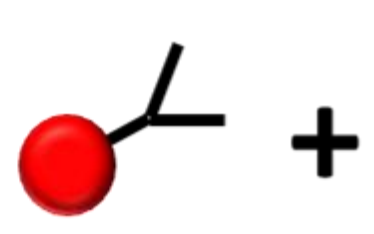

Antibody-modified magnetic nanoparticle

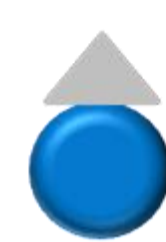

Cell with antigen

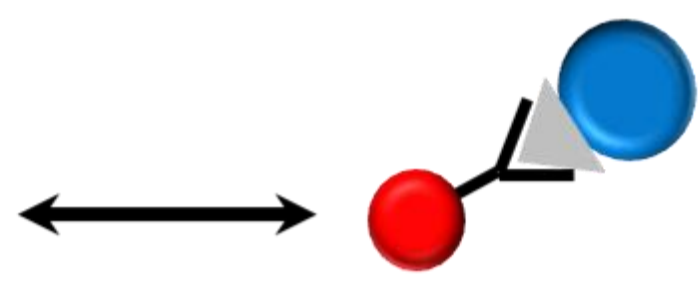

Magnetic nanoparticle Cell complex

Figure 3. Schematic of the binding reaction between the magnetic nanoparticle and bio cell using antigenantibody chemistry. 


\subsection{Continuous-flow microdevices based magnetophoresis.}

Various microchips configurations have been proposed and investigated for label-free particles in literature. Some of these configurations are shown in Figure 4, to illustrate the principle and work mechanism of the continuous flow microdevice-based magnetophorosis, pursuing to be applied for viruses detection and therapy purposes, specifically, COVID-19 diagnostics. Wherein, with the continuous flow of the IMBs suspension, under the effect of the applied external magnetic field, IMBs are separated from the fluid. Thus, the force for IMBs capture can be produced by using a micro magnet as shown in figure $4 \mathrm{a}$ and $\mathrm{b}$, or a prefabricated wire made from a ferromagnetic material; the wire can be switched on and off by simply applying and removing a bias field, thereby enabling the separation of IMBs from a sample and its subsequent release in higher concentration on demand for further analysis, The ferromagnetic wire can be positioned in the bottom of the microfluidic device, parallel to the flow direction for the separation of magnetic and non-magnetic nanoparticles as shown in figure $4 \mathrm{c}$ [27-30].

Khashan et al. [31,32] developed a multi-targets sorting microdevice, exclusively mediated by the repulsion force induced by a single ferromagnetic wire, as shown in Figure $4 \mathrm{~d}$ and e. Figure $4 \mathrm{~d}$ shows micro-wire is positioned flow-invasive through the depth of the microchannel transversal.

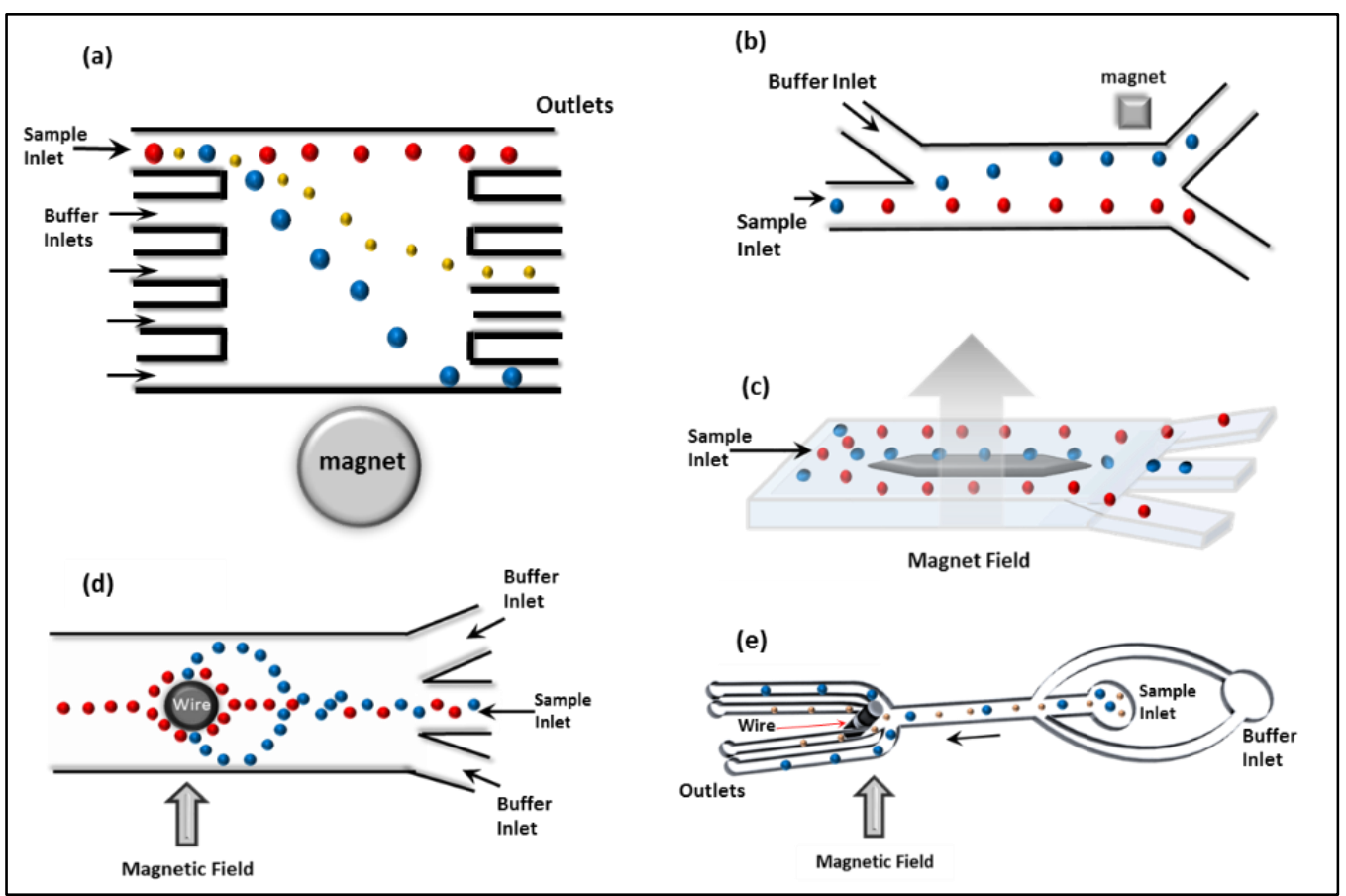

Figure 4. Schematic of continuous-flow magnetophoresis sorting of the mixture of non-magnetic entities and different sizes of magnetic nanoparticles in microdevices $(a, b)$ continuous separation of binary mixtures of magnetic particles based on size and non-magnetic entities, the permanent external magnet used for lateral displacement. (c) The ferromagnetic wire is positioned in the bottom of the microfluidic device, parallel to the flow direction for the separation of magnetic and non-magnetic nanoparticles. (d) Microchannel with hydrodynamically focused flow, and the wire embedded flow-invasive perpendicular in the center of the microchannel, demonstrate the trapping of magnetic nanoparticles and the elution of non-magnetic entities in the presence of the magnet field. (e) Size based continuous separation of binary mixtures of magnetic nanoparticles, the wire embedded perpendicular noninvasive, at the end of the microchannel. Herein, the arrows represent the flow direction, blue spheres denote the big magnetic nanoparticles, yellow spheres denote the small magnetic nanoparticles and red spheres denote non-magnetic entities [32]. (e) "Adapted from Microfluidics and Nanofluidics, 22, 6, Khashan, S.; Dagher, S.; Alazzam, A., Microfluidic multi-target sorting by magnetic repulsion, 64, 2018, with permission from Springer Nature.”. 
Opposite to the thinly focused sample flow, water is pumped from one inlet. It branches into two streams before entering the main microchannel from two opposing sides. The sample, carrying the magnetic nanoparticles, is pumped from another inlet. Once it enters the main microchannel, it is subjected to hydrodynamic focusing, by which it is squeezed into a thin sheet to approach the wire from its magnetically repulsive side. Specifically, the magnetic field is applied perpendicular to the wire. The magnetic nanoparticles trapped on the wire can be released back into the fluid by removing the external magnets, i.e., once the wire returns to its un-magnetized state. It is thereby enabling the separation of magnetic particles from a sample and its subsequent release in higher concentration on demand for further analysis.

Figure $4 \mathrm{e}$ shows the adapted design from Khashan et al. [32] indicating continuousflow, magnetophoresis-based microfluidic systems aimed at multi-target sorting. The wire is positioned noninvasive and perpendicular to the flow direction. Its long axis can span the microchannel's whole depth and is embedded just outside the main microchannel near its closed downstream end. The system arrangement is such that the magnetic field was adjusted to be perpendicular to the ferromagnetic wire and the flow direction. Thus, when the external magnetic field magnetizes the wire, the non-uniform magnetic field is present throughout the microchannel height, which allows for handling high throughput. The magnetic targets are repelled from the low gradient region towards the high gradient region. Accordingly, each target detaches from its laminar flow path and therefore separates from the focused layer towards a spatially addressable side microchannel. The throughput is scalable with the depth of the microchannel.

\section{Conclusions}

COVID-19 is an infectious disease caused by large seafood and live animal market in China, which promoted the animal-to-person spread. Later on, a growing number of patients reportedly who did not have exposure to animal markets were found to be infected, indicating the high affinity of person-to-person spread. Described sicknesses have ranged from very minor to severe. Death cases have also been reported. While the evidence so far suggests that most COVID-19 sicknesses are mild, public health and healthcare systems may become overloaded. The quality of services will reduce increasing rates of deaths. The world is not prepared for a medical crisis. Until this moment, there is no proof of treatment or vaccines that could effectively terminate the SARS-CoV-2. The comprehensive medical picture concerning COVID-19 remains unknown, and the spread is inevitable at this point. Thus, more studies are needed on the SARS-CoV-2 structure, RNA genome, enzymes, receptor-binding domain (RBD), and the host cell's entry mechanism. A deep understanding of the virus's structure and its hosting mechanism in the human body is essential to develop an effective vaccine. Therefore, several configurations of current available continuous flow microdevices-based magnetophoroses are discussed that might be used to open up new avenues for simultaneous using different-size magnetic nanoparticles (i.e., after modification into IMBs to detect multiple virus subtypes simultaneously. These microdevices are useful tools used to separate, enrich, react, and detect target viruses, point of care diagnostics, and other testing and analysis for therapy purposes, opening the way to be applied SARS-CoV-2 detection as a possible effective diagnostic method for the future.

\section{Funding}

This research received no external funding. 


\section{Acknowledgments}

The authors would like to thank the Abu Dhabi Polytechnic for their support of the study.

\section{Conflicts of Interest}

The authors declare no conflict of interest.

\section{References}

1. Marty, A.M.; Jones, M.K. The novel Coronavirus (SARS-CoV-2) is a one health issue. One Health 2020, 9 , https://doi.org/10.1016/j.onehlt.2020.100123.

2. Read, J.M.; Bridgen, J.R.E.; Cummings, D.A.T.; Ho, A.; Jewell, C.P. Novel coronavirus 2019-nCoV: early estimation of epidemiological parameters and epidemic predictions. medRxiv 2020, https://doi.org/10.1101/2020.01.23.20018549.

3. Tang, B.; Wang, X.; Li, Q.; Bragazzi, N.L.; Tang, S.; Xiao, Y.; Wu, J. Estimation of the Transmission Risk of the 2019-nCoV and Its Implication for Public Health Interventions. Journal of Clinical Medicine 2020, 9, https://doi.org/10.3390/jcm9020462.

4. Li, Q.; Guan, X.; Wu, P.; Wang, X.; Zhou, L.; Tong, Y.; Ren, R.; Leung, K.S.M.; Lau, E.H.Y.; Wong, J.Y.; Xing, X.; Xiang, N.; Wu, Y.; Li, C.; Chen, Q.; Li, D.; Liu, T.; Zhao, J.; Liu, M.; Tu, W.; Chen, C.; Jin, L.; Yang, R.; Wang, Q.; Zhou, S.; Wang, R.; Liu, H.; Luo, Y.; Liu, Y.; Shao, G.; Li, H.; Tao, Z.; Yang, Y.; Deng, Z.; Liu, B.; Ma, Z.; Zhang, Y.; Shi, G.; Lam, T.T.Y.; Wu, J.T.; Gao, G.F.; Cowling, B.J.; Yang, B.; Leung, G.M.; Feng, Z. Early Transmission Dynamics in Wuhan, China, of Novel Coronavirus-Infected Pneumonia. New England Journal of Medicine 2020, 382, 1199-1207, https://doi.org/10.1056/NEJMoa2001316.

5. Wu, J.T.; Leung, K.; Leung, G.M. Nowcasting and forecasting the potential domestic and international spread of the 2019-nCoV outbreak originating in Wuhan, China: a modelling study. The Lancet 2020, 395, 689-697, https://doi.org/10.1155/2019/1386740.

6. Wang, N.; Li, S.-Y.; Yang, X.-L.; Huang, H.-M.; Zhang, Y.-J.; Guo, H.; Luo, C.-M.; Miller, M.; Zhu, G.; Chmura, A.A.; Hagan, E.; Zhou, J.-H.; Zhang, Y.-Z.; Wang, L.-F.; Daszak, P.; Shi, Z.-L. Serological Evidence of Bat SARS-Related Coronavirus Infection in Humans, China. Virologica Sinica 2018, 33, 104107, https://doi.org/10.1007/s12250-018-0012-7.

7. Cui, J.; Li, F.; Shi, Z.L.; Origin and Evolution of Pathogenic Coronaviruses. Nature Reviews Microbiology 2019, 17, 181-192, https://doi.org/10.1038/s41579-018-0118-9.

8. Fan, Y.; Zhao, K.; Shi, Z.L.; Zhou, P. Bat Coronaviruses in China. Viruses. 2019, 11, https://doi.org/10.3390/v11030210.

9. Gorbalenya, A.E.; Baker, S.C.; Baric, R.S.; de Groot, R.J.; Drosten, C.; Gulyaeva, A.A.; Haagmans, B.L.; Lauber, C.; Leontovich, A.M.; Neuman, B.W.; Penzar, D.; Perlman, S.; Poon, L.L.M.; Samborskiy, D.V.; Sidorov, I.A.; Sola, I.; Ziebuhr, J.; Coronaviridae Study Group of the International Committee on Taxonomy of, V. The species Severe acute respiratory syndrome-related coronavirus: classifying $2019-\mathrm{nCoV}$ and naming it SARS-CoV-2. Nature Microbiology 2020, 5, 536-544, https://doi.org/10.1038/s41564-020-0695$\mathrm{z}$.

10. The Editors of Encyclopaedia Britannica, Coronavirus, Encyclopadia Britannica, 2020. https://www.britannica.com/science/coronavirus-virus-group.

11. Han Q.; Lin, Q.; Jin, S.; You, L.; Coronavirus 2019-nCoV: A brief perspective from the front line. Journal of Infection 2020, 80, 373-377, https://doi.org/10.1016/j.jinf.2020.02.010.

12. Zhou, P.; Yang, X.-L.; Wang, X.-G.; Hu, B.; Zhang, L.; Zhang, W.; Si, H.-R.; Zhu, Y.; Li, B.; Huang, C.L.; Chen, H.-D.; Chen, J.; Luo, Y.; Guo, H.; Jiang, R.-D.; Liu, M.-Q.; Chen, Y.; Shen, X.-R.; Wang, X.; Zheng, X.-S.; Zhao, K.; Chen, Q.-J.; Deng, F.; Liu, L.-L.; Yan, B.; Zhan, F.-X.; Wang, Y.-Y.; Xiao, G.-F.; Shi, Z.-L. A pneumonia outbreak associated with a new coronavirus of probable bat origin. Nature 2020, 579, 270-273, https://doi.org/10.1038/s41586-020-2012-7.

13. Xiao, K.; Zhai, J.; Feng, Y.; Zhou, N.; Zhang, X.; Zou, J.-J.; Li, N.; Guo, Y.; Li, X.; Shen, X.; Zhang, Z.; Shu, F.; Huang, W.; Li, Y.; Zhang, Z.; Chen, R.-A.; Wu, Y.-J.; Peng, S.-M.; Huang, M.; Xie, W.-J.; Cai, Q.H.; Hou, F.-H.; Liu, Y.; Chen, W.; Xiao, L.; Shen, Y. Isolation and Characterization of 2019-nCoV-like Coronavirus from Malayan Pangolins. bioRxiv 2020, https://doi.org/10.1101/2020.02.17.951335.

14. Tang, X.; Wu, C.; Li, X.; Song, Y.; Yao, X.; Wu, X.; Duan, Y.; Zhang, H.; Wang, Y.; Qian, Z.; Cui, J.; Lu, J. On the Origin and Continuing Evolution of SARS-CoV-2. National Science Review 2020, 7, 1012-1023, https://doi.org/10.1093/nsr/nwaa036.

15. Zheng, J. SARS-CoV-2: an Emerging Coronavirus that Causes a Global Threat. International Journal of Biological Sciences 2020, 16, 1678-1685, https://doi.org/10.7150/ijbs.45053. 
16. Li, W.; Moore, M.J.; Vasilieva, N.; Sui, J.; Wong, S.K.; Berne, M.A.; Somasundaran, M.; Sullivan, J.L.; Luzuriaga, K.; Greenough, T.C.; Choe, H.; Farzan, M. Angiotensin-converting enzyme 2 is a functional receptor for the SARS coronavirus. Nature 2003, 426, 450-454, https://doi.org/10.1038/nature02145.

17. Hofmann, H.; Pyrc, K.; van der Hoek, L.; Geier, M.; Berkhout, B.; Pöhlmann, S. Human coronavirus NL63 employs the severe acute respiratory syndrome coronavirus receptor for cellular entry. Proceedings of the National Academy of Sciences 2005, 102, 7988-7993, https://doi.org/10.1073/pnas.0409465102.

18. Xu, X.; Chen, P.; Wang, J.; Feng, J.; Zhou, H.; Li, X.; Zhong, W.; Hao, P. Evolution of the novel coronavirus from the ongoing Wuhan outbreak and modeling of its spike protein for risk of human transmission. Science China Life Sciences 2020, 63, 457-460, https://doi.org/10.1007/s11427-020-1637-5.

19. Zou, X.; Chen, K.; Zou, J.; Han, P.; Hao, J.; Han, Z. Single-cell RNA-seq data analysis on the receptor ACE2 expression reveals the potential risk of different human organs vulnerable to 2019-nCoV infection. Frontiers of Medicine 2020, 14, 185-192, https://doi.org/10.1007/s11684-020-0754-0.

20. Wang, M.; Cao, R.; Zhang, L.; Yang, X.; Liu, J.; Xu, M.; Shi, Z.; Hu, Z.; Zhong, W.; Xiao, G. Remdesivir and chloroquine effectively inhibit the recently emerged novel coronavirus (2019-nCoV) in vitro. Cell Research 2020, 30, 269-271, https://doi.org/10.1038/s41422-020-0282-0.

21. Motoo, Y. Antiproteases in the Treatment of Chronic Pancreatitis. Journal of the pancreas 2007, 8, 533-537.

22. Sheahan, T.P.; Sims, A.C.; Leist, S.R.; Schäfer, A.; Won, J.; Brown, A.J.; Montgomery, S.A.; Hogg, A.; Babusis, D.; Clarke, M.O.; Spahn, J.E.; Bauer, L.; Sellers, S.; Porter, D.; Feng, J.Y.; Cihlar, T.; Jordan, R.; Denison, M.R.; Baric, R.S. Comparative therapeutic efficacy of remdesivir and combination lopinavir, ritonavir, and interferon beta against MERS-CoV. Nature Communications 2020, 11, 222, https://doi.org/10.1038/s41467-019-13940-6.

23. Zhuang, J.; Yin, J.; Lv, S.; Wang, B.; Mu, Y. Advanced "Lab-on-a-chip" to Detect Viruses - current Challenges and Future Perspectives. Biosensors and Bioelectronics 2020, 163, https://doi.org/10.1016/j.bios.2020.112291.

24. Wang, S.; Ai, Z.; Zhang, Z.; Tang, M.; Zhang, N.; Liu, F.; Han, G.; Hong, S.-L.; Liu, K. Simultaneous and automated detection of influenza A virus hemagglutinin $\mathrm{H} 7$ and $\mathrm{H} 9$ based on magnetism and size mediated microfluidic chip. Sensors and Actuators B: Chemical 2020, 308, https://doi.org/10.1016/j.snb.2020.127675.

25. Zhang, R.-Q.; Hong, S.-L.; Wen, C.-Y.; Pang, D.-W.; Zhang, Z.-L. Rapid detection and subtyping of multiple influenza viruses on a microfluidic chip integrated with controllable micro-magnetic field. Biosensors and Bioelectronics 2018, 100, 348-354, https://doi.org/10.1016/j.bios.2017.08.048.

26. Wang, L.; Lin, J. Recent advances on magnetic nanobead based biosensors: From separation to detection. TrAC Trends in Analytical Chemistry 2020, 128, https://doi.org/10.1016/j.trac.2020.115915.

27. Alnaimat, F.; Dagher, S.; Mathew, B.; Hilal-Alnqbi, A.; Khashan, S. Microfluidics Based Magnetophoresis: A Review. The Chemical Record 2018, 18, 1596-1612, https://doi.org/10.1002/tcr.201800018.

28. Mathew, B., Hilal-Alnaqbi, A., Alazzam, A., Dagher, S., Analysis of Dielectrophoresis Based 3D-Focusing in Microfluidic Devices with Planar Electrodes, 39th Annual International Conference of the IEEE Engineering in Medicine and Biology Society, 11-15 July (2017), Jeju Island, Korea.

29. Khashan, S., Alazzam, A., Mathew, B., Dagher, S., Hamdan, M., CFD Simulation of Magnetic Separation in Microfluidics Systems Using Mixture Model, Eleventh International Conference on CFD in the Minerals and Process Industries CSIRO, 7-9 December (2015), Melbourne, Australia.

30. Mathew, B.; Alazzam, A,; Khashan, S.; Stiharu, I.; Dagher, S.; Furlani, E. Fabrication of Microfluidic Device with 3D Embedded Flow-invasive Microelements. Microelectronic Engineering 2018, 187-188, 27-32, https://doi.org/10.1016/j.mee.2017.11.013.

31. Khashan, S.; Dagher, S.; Alazzam, A.; Mathew, B.; Hilal-Alnaqbi, A. Microdevice for Continuous Flow Magnetic Separation for Bioengineering Applications. Journal of Micromechanics and Microengineering 2017, 27, https://doi.org/10.1088/1361-6439/aa666d.

32. Khashan, S.A.; Dagher, S.; Alazzam, A. Microfluidic multi-target sorting by magnetic repulsion. Microfluidics and Nanofluidics 2018, 22, https://doi.org/10.1007/s10404-018-2083-z. 